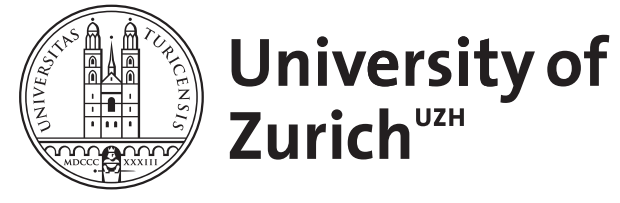

\title{
Schlafstörungen im Lebenszyklus
}

Zumsteg, Urs ; Suter, Paolo M

DOI: https://doi.org/10.1024/1661-8157/a003183

Posted at the Zurich Open Repository and Archive, University of Zurich ZORA URL: https://doi.org/10.5167/uzh-170462

Journal Article

Published Version

Originally published at:

Zumsteg, Urs; Suter, Paolo M (2019). Schlafstörungen im Lebenszyklus. Praxis, 108(2):87-88.

DOI: https://doi.org/10.1024/1661-8157/a003183 


\title{
Schlafstörungen im Lebenszyklus
}

\author{
Urs Zumsteg und Paolo M. Suter
}

Die Schlafmedizin ist innerhalb der Pädiatrie eine noch relativ junge, aber doch rasch wachsende Subspezialität, und gerade für den wachsenden Organismus ist ausreichender und erholsamer Schlaf von grosser Bedeutung. Schlafstörungen bei Kindern und Jugendlichen bedürfen einer hoch differenzierten und meist interdisziplinären Beurteilung. Einige dieser Störungen klingen mit zunehmenden Alter in der Regel ab, andere hingegen können ohne Therapie zu schwerwiegenden Folgeerkrankungen bis hin ins Erwachsenenalter führen. Wie fast immer in der Pädiatrie bedürfen die entwicklungs- und reifungsbedingten Besonderheiten unterschiedlicher Normwerte für die verschiedenen Altersstufen, das Wissen der altersabhängigen Physiologie ist damit auch in der pädiatrischen Schlafmedizin unabdingbare Voraussetzung für die Betreuung. Besondere Bedeutung haben Hyposomnien und Parasomnien, das grosse Spektrum der schlafbezogenen Atmungsstörungen sowie die schlafbezogenen, meist rhythmischen Bewegungsstörungen, im Weiteren müssen differenzialdiagnostisch Anfallsleiden, Funktionsanomalien des ZNS sowie psychiatrische Störungen ausgeschlossen werden. Es gilt, die «Red Flags» zu erkennen und die notwendigen Abklärungsschritte einzuleiten. Der technische und personelle Aufwand ist in der pädiatrischen Schlafmedizin klar erhöht, und je nach prädisponierender Grunderkrankung sind interdisziplinäre Therapieansätze unumgänglich. Und bei allen diagnostischen und therapeutischen Massnahmen müssen die Eltern ganz wesentlich miteinbezogen werden, nur so kann die Langzeitbetreuung unter Berücksichtigung der soziokulturellen Aspekte umfassend und zielführend sein.

Dieses Heft präsentiert zunächst pädiatrische Aspekte der Schlafmedizin aus pädiatrisch-pneumologischer und neuropädiatrischer Sicht $[1,2]$, die beiden klinisch und wissenschaftlich sehr erfahrenen Autoren präsentieren darüber hinaus aber den ganzheitlichen Ansatz und das interdisziplinäre Betreuungskonzept in der Schlafmedizin bei Kindern und Jugendlichen.

Ein gesunder Schlaf im Kindesalter ist die Voraussetzung für einen erfolgreichen Übergang und Eintritt ins Erwachsenenalter. Schlafstörungen bei Erwachsenen haben oft ihre Ursache in der Kindheit und beinhalten ein hohes Risiko der Persistenz und Intensivierung derselben im Adoleszenten- und Erwachsenenalter [3]. Dem Lebenszyklus folgend, werden im zweiten Teil des Heftes ausgewählte Schlafthemen aus der Erwachsenenmedizin erörtert und zusammengefasst, wobei wir aufgrund der Komplexität und Dynamik der Schlafforschung Schwerpunkte legen mussten: vom Screening [4], über Schlaf bei Schichtarbeitern [5] und im Alter [6] bis hin zum obstruktiven Schlafapnoe-Syndrom [7] und zur modernen Phar- makotherapie von Schlafstörungen [8]. Die Bedeutung des modernen Lebensstils in der Pathogenese von Schlafstörungen wird nur gestreift - sozusagen zwischen den Zeilen. Ein neues, zunehmend relevantes Phänomen soll aber hier nicht unerwähnt bleiben. Paradoxerweise sucht der Schlafpatient oftmals initiale Hilfe in der "Consumer Sleep Technology» (CST, wie z.B. Sleep Trackers) $[9,10]$ und nicht bei seinem Hausarzt. Diese Geräte können allerdings eine neue, bisher nicht bekannte Schlafstörung, die sogenannte «Orthosomnie», induzieren [11]. Das Selbst-Monitoring des Schlafs bei (mehr oder weniger) gesunden, nicht schlafgestörten oder nur situativ schlafgestörten Individuen kann den Schlaf und das Schlafverhalten negativ beeinflussen: Die CST induziert und verstärkt die Angst vor einer möglichen Schlafstörung, die dann schlussendlich in einer Schlafstörung resultiert. Wir wissen seit Langem, dass ein übertriebenes SelbstTracking und -Monitoring ein hohes Krankheitspotenzial birgt: Wie sagte doch schon Laotse: «Nur das kranke Auge sieht sich selber.» In diesem Sinne präsentieren wir in diesem Heft praxisrelevante Schlafthemen, sodass die Patienten der «Praxis»-Leser Schlafprobleme ohne CST in der hausärztlichen Sprechstunde angehen und lösen können.

\section{Bibliografie}

1. Datta AN, Trachsel D: Der kindliche Schlaf: ein Buch mit sieben Siegeln? Eine Anleitung zur systematischen Problemerkennung und -lösung. Praxis 2019;108: 89-95.

2. Trachsel D, Datta AN: Atmen im Schlaf - nicht immer leicht gemacht. Praxis 2019; 108: 97-102

3. Sivertsen B, Harvey AG, Pallesen S, Hysing M: Trajectories of sleep problems from childhood to adolescence: A populationbased longitudinal study from Norway. J Sleep Res 2017; 26: 55-63.

4. Acker JG, Cordi MJ: Schlafprobleme in der Allgemeinpraxis: Vom Screening zur initialen Therapie - Update 2019. Praxis 2019; 108: 103-109.

5. Hicklin D, Schwander J: Schichtarbeit und Schlaf. Praxis 2019; 108:119-124.

6. Flügel D: Schlafstörungen im Alter. Praxis 2019;108:125-130.

7. Stöwhas AC, Lichtblau M, Bloch KE: Obstruktives Schlafapnoe-Syndrom. Praxis 2019; 108:111-117.

8. Holst SC, Werth E, Landolt HP: Pharmakotherapie von SchlafWach-Störungen. Praxis 2019; 108:131-138.

9. Khosla S, Deak MC, Gault D, et al.: Consumer sleep technology: An American Academy of sleep medicine position statement. $J$ Clin Sleep Med 2018; 14:877-880.

10. Suter PM: Mach nicht zu viel «blau». Gedanken zu Licht und Schlaf. 139-143.

11. Baron KG, Abbott S, Jao N, Manalo N, Mullen R: Orthosomnia: Are some patients taking the quantified self too far? J Clin Sleep Med 2017: 13: 351-354 


\section{Prof. Dr. med. Urs Zumsteg}

Chefarzt Ambulante Medizin

Pädiatrische Endokrinologie/Diabetologie

Universitäts-Kinderspital beider Basel (UKBB)

Spitalstrasse 33 Postfach

4031 Basel

urs.zumsteg@ukbb.ch

\section{Prof. Dr. med. Paolo M. Suter}

Leitender Arzt

Klinik und Poliklinik für Innere Medizin

Universitätsspital

8991 Zürich

paolo.suter@usz.ch 